OPEN ACCESS

Edited by:

Cheng Zhong,

Tianjin University, China

Reviewed by:

Wenhui Zeng,

Procter and Gamble, United States

Hao Wang,

National Renewable Energy Laboratory (DOE), United States

*Correspondence: Lei Zhang

leizhang@nwpu.edu.cn Hong Zhong

zhonghong123@nwpu.edu.cn

Specialty section: This article was submitted to

Electrochemistry,

a section of the journal

Frontiers in Chemistry

Received: 30 July 2021 Accepted: 31 August 2021 Published: 01 October 2021

Citation:

Liu Z, Bu J, Ma W, Yang B, Zhang L, Zhong H, Li S and Zhang J (2021) Regulating Water Reduction Kinetics on MoP Electrocatalysts Through Se

Doping for Accelerated Alkaline

Hydrogen Production.

Front. Chem. 9:737495.

doi: 10.3389/fchem.2021.737495

\section{Regulating Water Reduction Kinetics on MoP Electrocatalysts Through Se Doping for Accelerated Alkaline Hydrogen Production}

\author{
Zhenpeng Liu ${ }^{1,2}$, Jun $\mathrm{Bu}^{2}$, Wenxiu $\mathrm{Ma}^{2}$, Bin Yang ${ }^{1}$, Lei Zhang ${ }^{2 *}$, Hong Zhong ${ }^{1 *}$, \\ Shuangming $L i^{1}$ and Jian Zhang ${ }^{2}$
}

${ }^{1}$ State Key Laboratory of Solidification Processing, Northwestern Polytechnical University, Xi'an, China, ${ }^{2}$ Key Laboratory of Special Functional and Smart Polymer Materials of Ministry of Industry and Information Technology and Department of Advanced Chemical Engineering, School of Chemistry and Chemical Engineering, Northwestern Polytechnical University, Xi'an, China

Owing to its low cost, high conductivity, and chemical stability, Molybdenum phosphide (MoP) has great potential for electrochemically catalyzing the hydrogen evolution reaction (HER). Unfortunately, the development of high-activity MoP still remains a grand challenge in alkali-electrolyzers due to its sluggish water reduction kinetics. Here, we demonstrate a novel strategy for regulating the HER kinetics of the MoP nanowire cathode through partially substituting $P$ atoms with Se dopants. In alkaline solutions, the Se-doped MoP (Se-MoP) nanowire cathode exhibits excellent HER performance with a greatly-decreased overpotential of $\sim 61 \mathrm{mV}$ at $10 \mathrm{~mA} \mathrm{~cm}{ }^{-2}$ and a Tafel slope of $\sim 63 \mathrm{mV} \mathrm{dec}^{-1}$, outperforming currently reported MoP-based electrocatalysts. Experimental and theoretical investigations reveal that Se doping not only facilitates the water dissociation on MoP, but also optimize the hydrogen adsorption free energy, eventually speeding up the sluggish alkaline HER kinetics. Therefore, this work paves a new path for designing MoP-based electrocatalyst with high HER performance in alkaline electrolyzers.

Keywords: MoP nanowires, Se doping, water-reduction kinetics, electrocatalytic, hydrogen evolution reaction

\section{INTRODUCTION}

Because of its large energy density and zero carbon emission, hydrogen $\left(\mathrm{H}_{2}\right)$ has been proposed as the most promising energy carrier for meeting future energy demands (Turner, 2004; Chu and Majumdar, 2012; Chu et al., 2017). Electrochemical water splitting to generate hydrogen is an efficient and sustainable pathway in the future hydrogen economy cycle (Norskov and Christensen, 2006; Seh et al., 2017). Nevertheless, owing to the large energy barrier of water dissociation, exploring highly efficient electrocatalysts is highly desirable for intrinsically improving the reaction kinetics and reducing HER overpotential, especially in alkaline media (Chen et al., 2018; Wang et al., 2020b; $\mathrm{Zhu}$ et al., 2020). To date, noble metal-based catalysts ( $\mathrm{Pt}, \mathrm{Ru}$, etc) are still the benchmark electrocatalysts because of their superior intrinsic HER activity (Lei et al., 2020; Yu et al., 2020). Unfortunately, the expensiveness and rareness of noble metals seriously limit its large-scale utilization in practical electrolyzers (Fang et al., 2020; Xue et al., 2020).

Recently, transition metal phosphides (TMPs), such as MoP show appealing HER performance among various HER electrocatalysts owing to their high conductivity, chemical stability, and similar electronic structure to Pt (Xiao et al., 2014; Yang et al., 2016; Zhang et al., 2018b; Song et al., 2020). 
Nevertheless, HER activity of MoP is still unsatisfactory compared with those of noble metal-based catalysts, especially in alkaline electrolytes (Jaramillo et al., 2007; Xing et al., 2014; Shi and Zhang, 2016). Therefore, improving the activity and stability of MoP catalysts is urgently demanded in alkaline solutions. Principally, the alkaline HER reaction proceeds via an initial Volmer step $\left(\mathrm{H}_{2} \mathrm{O}+\mathrm{e}^{-} \rightarrow \mathrm{H}^{*}+\mathrm{OH}^{-}\right)$and a sequent Heyrovsky step $\left(\mathrm{H}_{2} \mathrm{O}+\mathrm{e}^{-}+\mathrm{H}^{*} \rightarrow \mathrm{H}_{2}+\mathrm{OH}^{-}\right)$or Tafel step $\left(\mathrm{H}^{*}+\mathrm{H}^{*} \rightarrow \mathrm{H}_{2}\right)$ (Strmcnik et al., 2016; Wang et al., 2020a). High energy barrier of water dissociation and unamiable hydrogen free energy on MoP catalysts leads to their poor HER performance (Subbaraman et al., 2012; Xu et al., 2017; Chen et al., 2018; Kim et al., 2021). Anion doping has been proven to be a useful method to improve the HER performance of MoP electrocatalysts, including the S-doped MoP nanoporous layer (Liang et al., 2019) and N-doped MoP nanoparticle catalyst (Chen et al., 2020). However, in previous reports, only the water dissociation energy barrier or $\mathrm{H}^{\star}$ adsorption energy was individually considered to play a critical role in promoting the HER reaction kinetics (Kibsgaard and Jaramillo, 2014; Anjum and Lee, 2017; Liang et al., 2019; Chen et al., 2020). In fact, some electrocatalysts have been designed to reduce both water dissociation energy barrier and $\mathrm{H}$ adsorption energy for the enhancement of HER performance, including $\mathrm{Ni}(\mathrm{OH})_{2} / \mathrm{NiMoPO}_{\mathrm{x}}$ (Peng et al., 2020), $\mathrm{Ni}$-doped $\mathrm{MoS}_{2}$ (Zhang et al., 2016), oxygen-incorporating $\mathrm{Co}_{2} \mathrm{P}$ (Xu et al., 2017), Cr-doped $\mathrm{Co}_{4} \mathrm{~N}$ (Yao et al., 2019), etc. Nevertheless, to our best knowledge, it is rarely reported to simultaneously reduce the water-dissociation and hydrogen adsorption energies using anion-doped MoP catalysts toward the HER. In addition, the conventional powdery catalysts should be coated on the current collector using polymeric binder, leading to the inferior conductivity, limited active sites and weak interactions between the catalyst and support (Zhang et al., 2018a; Zhang et al., 2018c; Huang et al., 2019). Accordingly, binder-free MoP could be served as an ideal electrode with high HER performance.

In this work, we develop a phosphorization-selenization process to synthesize Se-doped MoP nanowires consisting of nanoparticles using ultralong Mo nanowires as the Mo source. The Se-MoP nanowires were assembled into a self-supported cathode and performed an excellent HER activity with a low overpotential of $\sim 61 \mathrm{mV}$ at $10 \mathrm{~mA} \mathrm{~cm}^{-2}$ and a small Tafel slope of $\sim 63 \mathrm{mV} \mathrm{dec}^{-1}$ in $1 \mathrm{M} \mathrm{KOH}$ solution, which were superior than pure $\mathrm{MoP}$ and previously reported $\mathrm{MoP}$-based electrocatalysts. Experimental investigations and theoretical calculations revealed that Se doping are beneficial for decreasing water dissociation energy and optimizing hydrogen adsorption energy, which ultimately enhanced the sluggish water-reduction kinetics for alkaline hydrogen evolution.

\section{EXPERIMENTAL DETAILS}

\section{Synthesis of Se-MoP Nanowire Film}

A two-temperature zone tube furnace was employed to prepare the Se-MoP nanowire film. The Mo nanowire film (more details in Supplementary Material) was placed into the downstream zone, while $\mathrm{NaH}_{2} \mathrm{PO}_{2}$ or selenium powder was placed in the upstream zone. To synthesize the MoP nanowire film, the temperature of upstream zone was heated up to $300^{\circ} \mathrm{C}$ and the temperature of the downstream zone was heated up to 800,900 , and $1000^{\circ} \mathrm{C}$, respectively. The fabrication processes were carried out for $1 \mathrm{~h}$ under a $\mathrm{N}_{2}$ atmosphere. When the temperatures were cooled to room temperature, the MoP nanowire film was obtained. The subsequent annealing treatment was performed using selenium powder to replace $\mathrm{NaH}_{2} \mathrm{PO}_{2}$, the temperature for selenium powder was $300^{\circ} \mathrm{C}$, and the temperature for the $\mathrm{MoP}$ nanowire film was $550^{\circ} \mathrm{C}$. To adjust the reaction time, Se-MoP nanowire film with different Se contents was obtained.

\section{Electrochemical Measurements}

Electrocatalytic HER activity of the prepared samples was measured in a CHI760E electrochemical workstation. Threeelectrode configuration was equipped with a graphite rod as the counter electrode, $\mathrm{Hg} / \mathrm{HgO}$ as the reference electrode and $\mathrm{MoP}$ and Se-MoP nanowire film as the working electrode. Liner sweep voltammetry (LSV) with a scan rate of $2 \mathrm{mV} \mathrm{s}^{-1}$ was conducted in a $1 \mathrm{M} \mathrm{KOH}$ aqueous solution. Electrochemical impendence spectroscopy (EIS) was carried out at $-0.1 \mathrm{~V}$ vs. RHE over a frequency range of $10 \mathrm{kHz}-0.01 \mathrm{~Hz}$. Cyclic voltammetry $(\mathrm{CV})$ measurements at different scan rates were performed to determine the electrochemically active specific area (ECSA). RHE potentials were calculated according to the equation: $\mathrm{E}_{\mathrm{RHE}}=\mathrm{E}_{\mathrm{Hg} / \mathrm{HgO}}+0.099 \mathrm{~V}+0.059 \mathrm{~V} \times \mathrm{pH}$.

\section{Theoretical Calculations}

The Vienna Ab Initio Simulation Package (VASP) code was adopted to carried out all density functional theory (DFT) calculations. (Kresse and Furthmüller, 1996a; Kresse and Furthmüller, 1996b). The Perdew-Burke-Ernzerhof (PBE) functional (Perdew et al., 1996) was used to describe the exchange correlation interaction by the generalized gradient approximation (GGA) method (Blöchl, 1994; Kresse and Joubert, 1999). The kinetic energy cut-off was set to $400 \mathrm{eV}$ and the convergences criteria for the residual forces and energies were set to $0.02 \mathrm{eV} / \AA$ and $10^{-5} \mathrm{eV}$, respectively. A vacuum layer of more than $15 \AA$ was set to avoid the interaction between the periodically repeated slabs. The reaction energetics for hydrogen evolution reaction intermediates were calculated using the computational hydrogen electrode model (Henkelman et al., 2000). For MoP (101) surface, a four-layer of $2 \star 3$ supercell was built during the calculation, and for Se doped MoP (101), one P atom on the top surface was doped by Se atom.

\section{RESULTS AND DISCUSSION}

Figure 1A schematically illustrated the fabrication procedure of Se-MoP nanowire film. First, ultralong Mo nanowires (diameter: $\sim 195 \mathrm{~nm}$, length: $>60 \mu \mathrm{m}$ ) were prepared from a directionally solidified NiAl-9Mo eutectic alloy by selective etching and then were assembled into a self-supported film through vacuum filtration (Supplementary Figure S1) (Liu et al., 2020). 
A

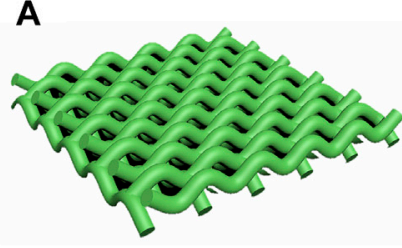

Mo nanowire film

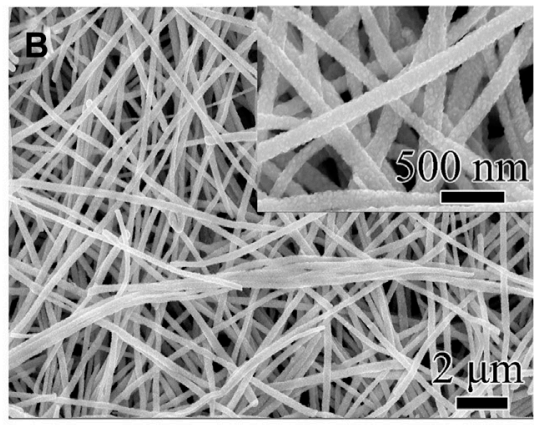

E
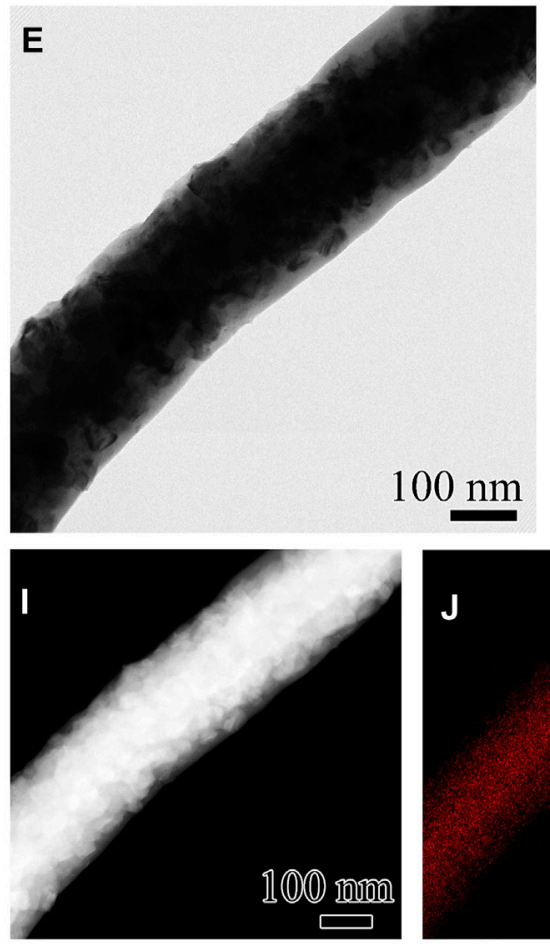

1. Phosphorization

2. Selenization

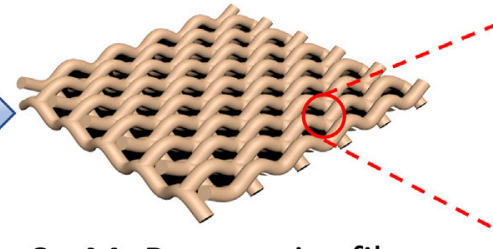

Se-MoP nanowire film
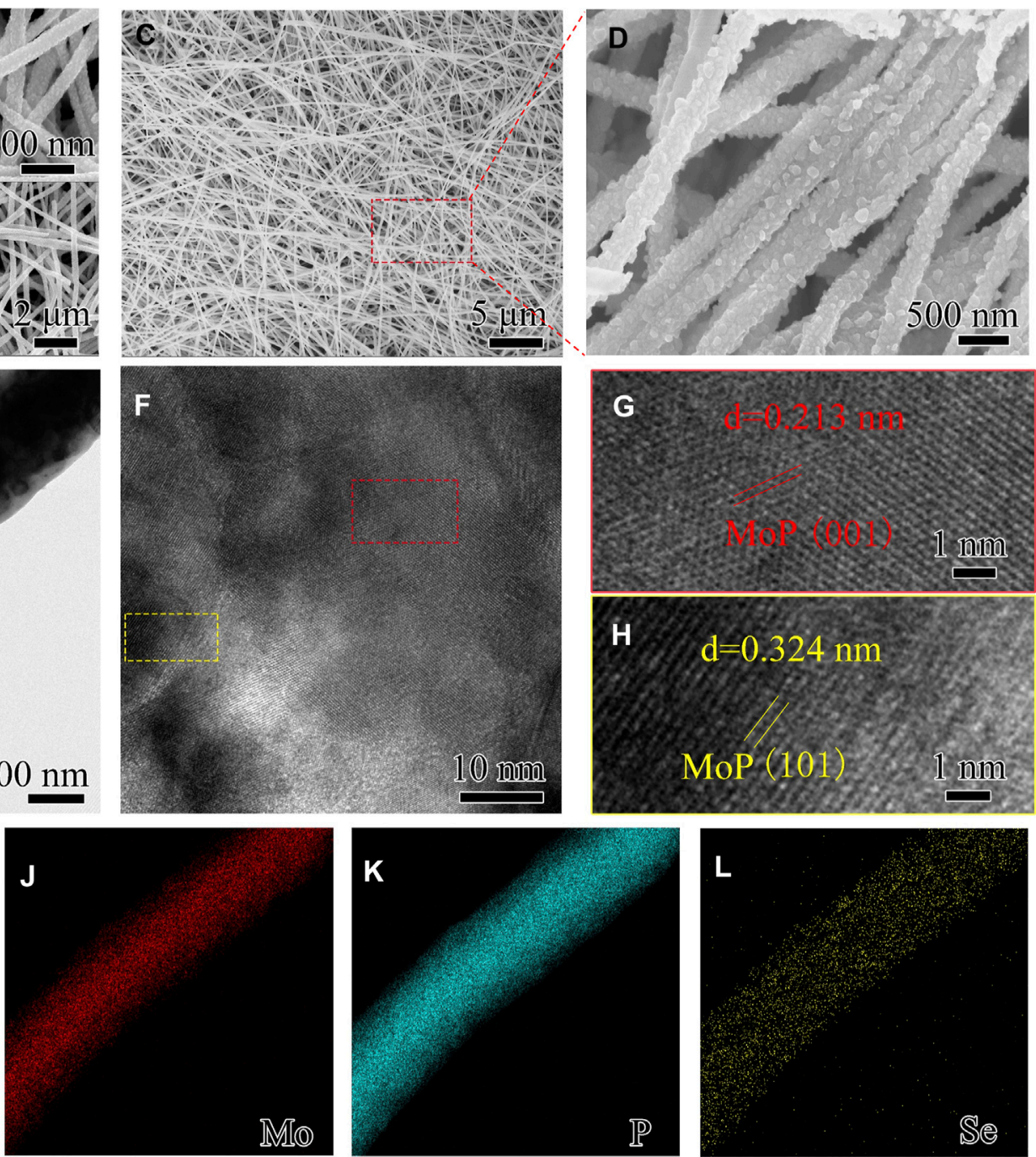

FIGURE 1 | (A) Synthetic route of the Se-MoP nanowire film. The blue, green, yellow, red, and white spheres represent Mo, P, Se, O, and H atoms, respectively. (B) SEM images of MoP nanowires and (C-D) Se-MoP nanowires. (E) TEM, (F-H) HRTEM, (I) HAADF-STEM images of the Se-MoP nanowires and the corresponding elemental mapping images of (J) Mo, (K) P, and (L) Se elements. The inset in Panel (B) represents corresponding high-magnification SEM image of MoP nanowires.

Second, MoP nanowires with sizes of $20-60 \mathrm{~nm}$ nanoparticles were obtained after phosphorization treatment at $900^{\circ} \mathrm{C}$ for $1 \mathrm{~h}$ using $\mathrm{NaH}_{2} \mathrm{PO}_{2}$ as a phosphorus source (Figure 1B, Supplementary Figures S2, S3). Eventually, after a half-hour annealing treatment of precursor $\mathrm{MoP}$ nanowire film at $550^{\circ} \mathrm{C}$ in Se vapor environments, the Se-MoP nanowire film was achieved. Se-doped MoP samples with different Se contents were prepared by changing selenization time.
The microstructures of $\mathrm{MoP}$ and Se-MoP nanowire electrocatalysts were evaluated by scanning electron microscopy (SEM) and transmission electron microscopy (TEM). As displayed in Figures 1C,D and Supplementary Figure S4, both MoP and Se-MoP nanowires were composed of nanoparticles with the size from 30 to $50 \mathrm{~nm}$. TEM image of $\mathrm{Se}-\mathrm{MoP}$ in Figure 1E further confirmed the nanowire structure with a larger diameter of $\sim 253 \mathrm{~nm}$ than the Mo nanowires, which 

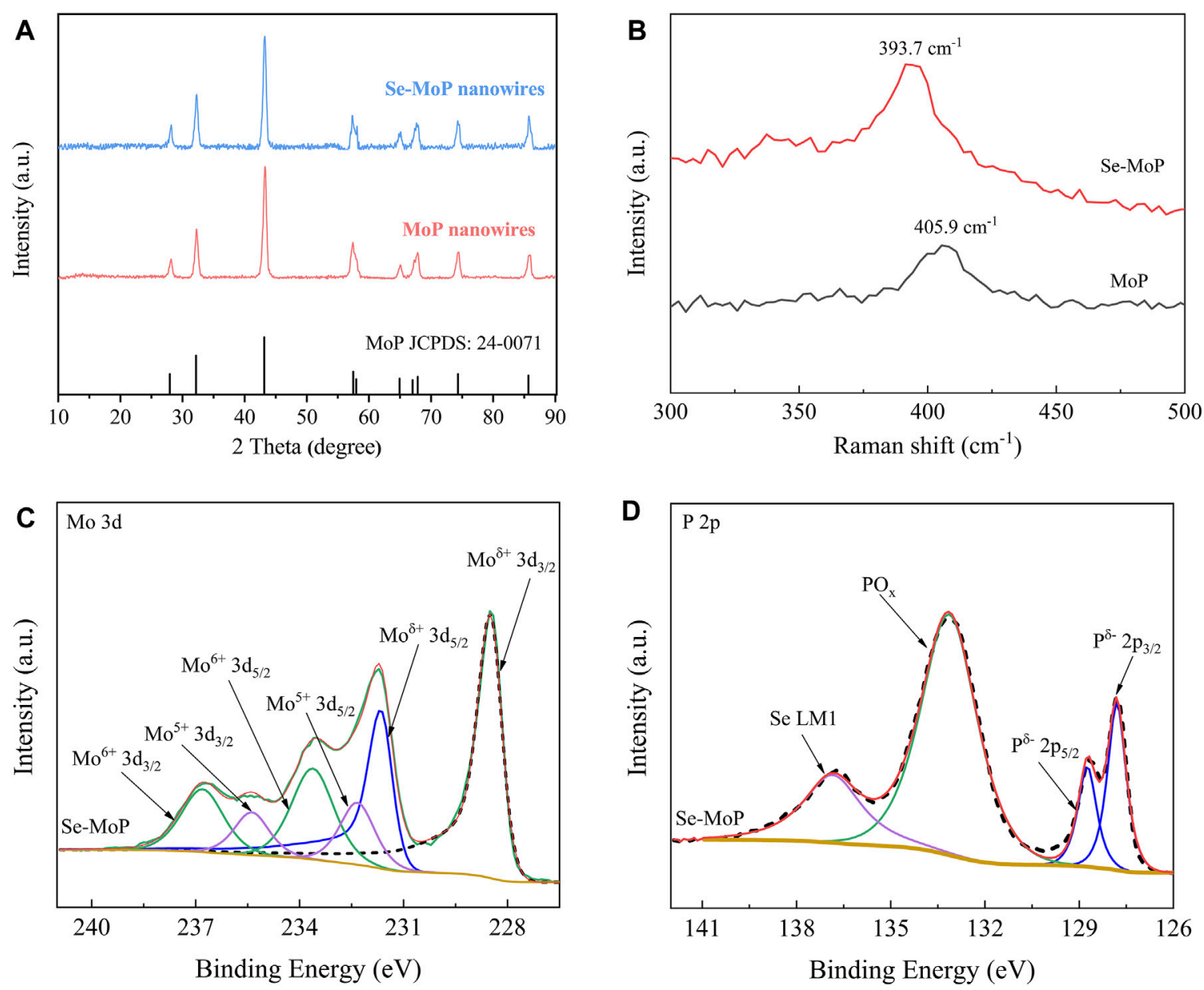

FIGURE 2 | (A) XRD patterns, (B) Raman spectra, (C) high-resolution XPS spectra of Mo 3 days, and (D) P 2p of Se-MoP nanowires.

was due to the growth of MoP. Subsequently, high-resolution TEM images show two interplanar spacings of 0.213 and $0.324 \mathrm{~nm}$ (Figures 1G,H), which are in accord with the (101) and (001) planes of MoP (Du et al., 2017; Xiao et al., 2020), respectively. The lattice space of Se-MoP nanowires was larger than the pure MoP (Supplementary Figure S5), indicating that the doping of Se could cause lattice expansion of MoP. Supplementary Figure S6 displays the diffraction patterns of the (001) and (101) facets of MoP. In addition, the elemental mapping of Se-MoP nanowires in Figure 1I demonstrates the uniform distribution of Mo, $\mathrm{P}$ and Se element. The Se content in Se-MoP nanowires (Se-MoP-30 min) was estimated to be $\sim 3.42$ at\% according to the EDX spectrum in Supplementary Figure S7. The morphologies of the Se-MoP nanowires under different selenization time were investigated. As disclosed in Supplementary Figure S8, all Se-MoP samples showed similar nanowire morphology. The atomic contents of Se in Se-MoP nanowires were found to be $\sim 1.27$ and $\sim 5.94$ at $\%$ (Supplementary Table S1) after 15-min (Se-MoP-15 min) and 60-min (Se-MoP-60 min) selenization.

The crystal information of the catalysts was evaluated by X-ray diffraction (XRD). As shown in Figure 2A and Supplementary
Figure S9A, for both $\mathrm{MoP}$ and Se-MoP nanowires, the diffraction peaks located at $27.9^{\circ}, 32.1^{\circ}, 43.2^{\circ}, 57.3^{\circ}, 57.7^{\circ}, 64.8^{\circ}, 67.6^{\circ}, 74.3^{\circ}$, and $85.7^{\circ}$ originated from the (001), (100), (101), (110), (002), (111), (102), (201), and (112) planes of the hexagonal MoP phase (JCPDS 24-0071) (Liu et al., 2018; Zhang et al., 2018b; Zhang et al., 2018c), respectively. Noticeably, no peaks of Se-related phases could be detected in the Se-MoP nanowires. Along with increased Se contents, the peak of the (101) facet shifted slightly to smaller 2- $\theta$ angles compared with pure MoP (Supplementary Figure S9B), implying that the Se doping caused lattice expansion of the MoP. This result agreed well with the HRTEM results. Then, the MoP and Se-MoP phases were further investigated through Raman spectroscopy. (Figure 2B). Clearly, the distinct peak of the stretching mode in Se-MoP at $393.7 \mathrm{~cm}^{-1}$ shifted to a low wavenumber compared to pure $\mathrm{MoP}\left(405.9 \mathrm{~cm}^{-1}\right.$ ) (Yin et al., 2016).

For investigating the valence state of un-doped and doped MoP electrocatalysts, X-ray photoelectron spectroscopy (XPS) was carried out. As shown in Supplementary Figure S10, the Mo, $\mathrm{P}$ and Se elements exist in Se-MoP nanowires. Figure 2C and Supplementary Figure S11 present the high-resolution spectra of the Mo $3 \mathrm{~d}$ peaks of the Se-MoP and MoP 

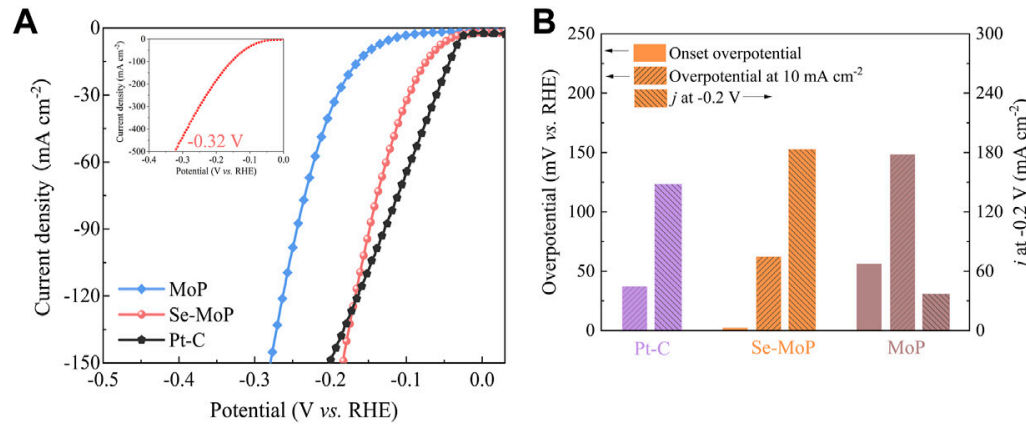

D
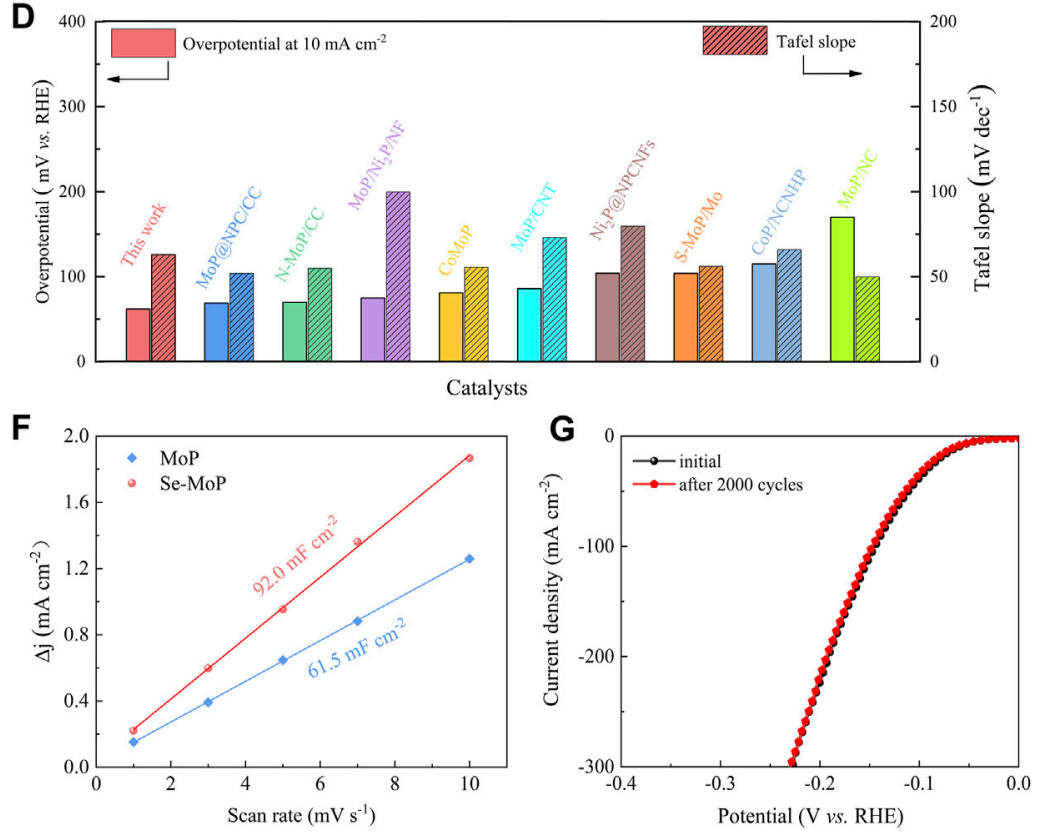
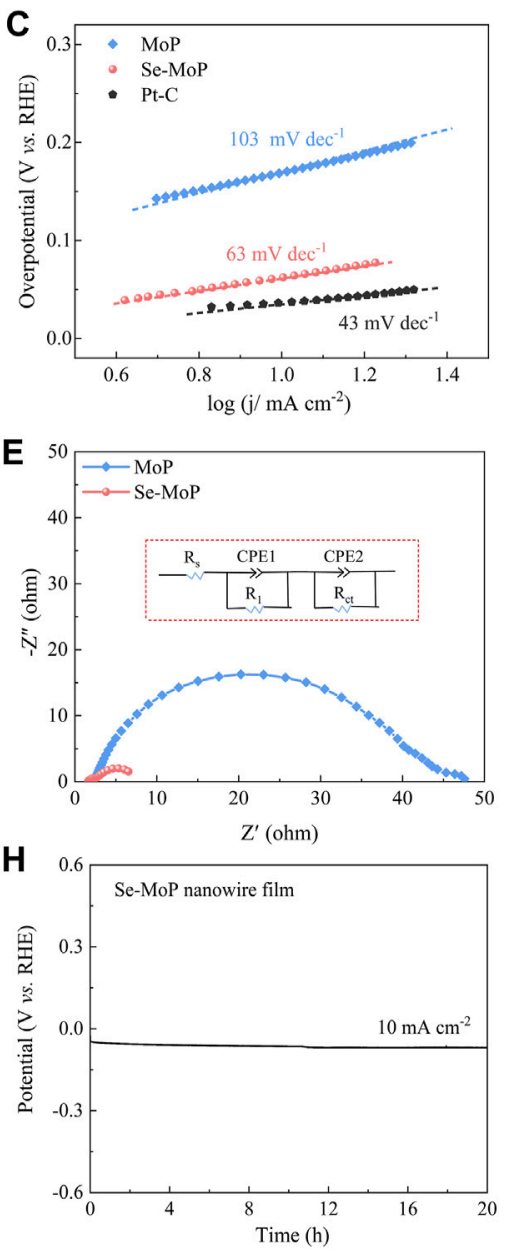

FIGURE 3 | Electrochemical investigations of Se-MoP nanowires, MoP nanowires and commercial Pt/C electrocatalysts in $1 \mathrm{M}$ KOH solution. (A) LSV curves. (B) Comparison of various catalysts in onset potential, overpotential at $10 \mathrm{~mA} \mathrm{~cm}{ }^{-2}$ and current densities at $-0.2 \mathrm{~V}$ vs. RHE. (C) The corresponding Tafel plots of various catalysts. (D) Comparison of the overpotential and Tafel slope among the Se-MoP nanowires and reported transition metal phosphate-based electrocatalysts under alkaline conditions. (E) Nyquist plots (-0.1 V vs. RHE) of MoP and Se-MoP nanowires in $1 \mathrm{M} \mathrm{KOH}$. (F) Double-layer capacitances. (G) LSV curves of Se-MoP nanowires before and after 2,000 cyclic voltammetry cycles. (H) Chronopotentiometric measurement of Se-MoP nanowires at $10 \mathrm{~mA} \mathrm{~cm}^{-2}$.

nanowires. The peaks of Se-MoP nanowires located at 228.5 and $231.7 \mathrm{eV}$, which were attributed to $\mathrm{Mo}^{\delta+}\left(3 \mathrm{~d}_{5 / 2}\right)(0<\delta<4)$ and $\left(3 d_{3 / 2}\right)$ of MoP (Chen et al., 2020; Xiao et al., 2020), respectively. The other two doublet peaks located at relatively higher binding energies of 232.3/235.4 and 233.6/236.9 eV were corresponding to $\mathrm{Mo}^{5+} 3 \mathrm{~d}_{5 / 2} / \mathrm{Mo}^{5+} 3 \mathrm{~d}_{3 / 2}$ and $\mathrm{Mo}^{6+} 3 \mathrm{~d}_{5 / 2} / \mathrm{Mo}^{6+} 3 \mathrm{~d}_{3 / 2}$, due to surface oxidation of Mo (Chen et al., 2017). The high-solution $\mathrm{P}$ $2 \mathrm{p}$ spectrum of Se-MoP in Figure 2D has two peaks at 129.7 and $130.6 \mathrm{eV}$, which originates from $\mathrm{p}^{\delta-}\left(3 \mathrm{p}_{3 / 2}\right)$ and $\mathrm{p}^{\delta-}\left(3 \mathrm{p}_{5 / 2}\right)$ of Mo-P bonds in MoP (Ye et al., 2016), respectively. The peak positioned at $134.7 \mathrm{eV}$ could be assigned to $\mathrm{PO}_{\mathrm{x}}$ species caused by slightly oxidized P (Liu et al., 2021). The Se-MoP nanowires displayed a peak at $138.7 \mathrm{eV}$, which could be derived from Se LM1. In the XPS Se 3d spectra (Supplementary Figure S12) of Se-MoP nanowires, peaks at 54.5 and $55.3 \mathrm{eV}$ were associated with Se $\left(3 d_{3 / 2}\right)$ and Se $\left(3 d_{5 / 2}\right)$ of Mo-Se bonds (Wang et al., 2014). The above SEM, TEM, XRD, Raman, and XPS results together demonstrate that the Se atoms were successfully doped into MoP nanowires with a uniform distribution by partially substituting $\mathrm{P}$ atoms.

The HER activity of MoP nanowire film and Se MoP nanowire film was assessed in a $1 \mathrm{M} \mathrm{KOH}$ solution. The commercial Pt/C loaded on a nickel foam was used as the reference catalyst. All LSV curves were iR compensated. As depicted in Figures 3A,B, the Se-MoP nanowire cathode exhibited an onset HER potential of $\sim 2 \mathrm{mV}$ and an overpotential of $\sim 61 \mathrm{mV}$ at $10 \mathrm{~mA} \mathrm{~cm}^{-2}$, which were much lower than pure MoP nanowires $(\sim 148 \mathrm{mV})$ and those for previously reported MoP-based HER electrocatalysts, such as N-doped MoP/CC ( 70 mV) (Chen et al., 2020), CoMoP@Ndoped C ( 81 mV) (Ma et al., 2017), S-doped MoP nanoporous layer $(\sim 104 \mathrm{mV})$ (Liang et al., 2019), carbon-coated Ni-doped MoP ( 162 mV) (Xiao et al., 2020), and MoP nanoparticles/NC $(\sim 170 \mathrm{mV})$ (Huang et al., 2019) (Supplementary Table S2). Apparently, the Se-MoP nanowire cathode achieved a very large current density of $184 \mathrm{~mA} \mathrm{~cm}^{-2}$ at $-0.2 \mathrm{~V}$, which was approximately 4.95 times higher than that for the MoP 

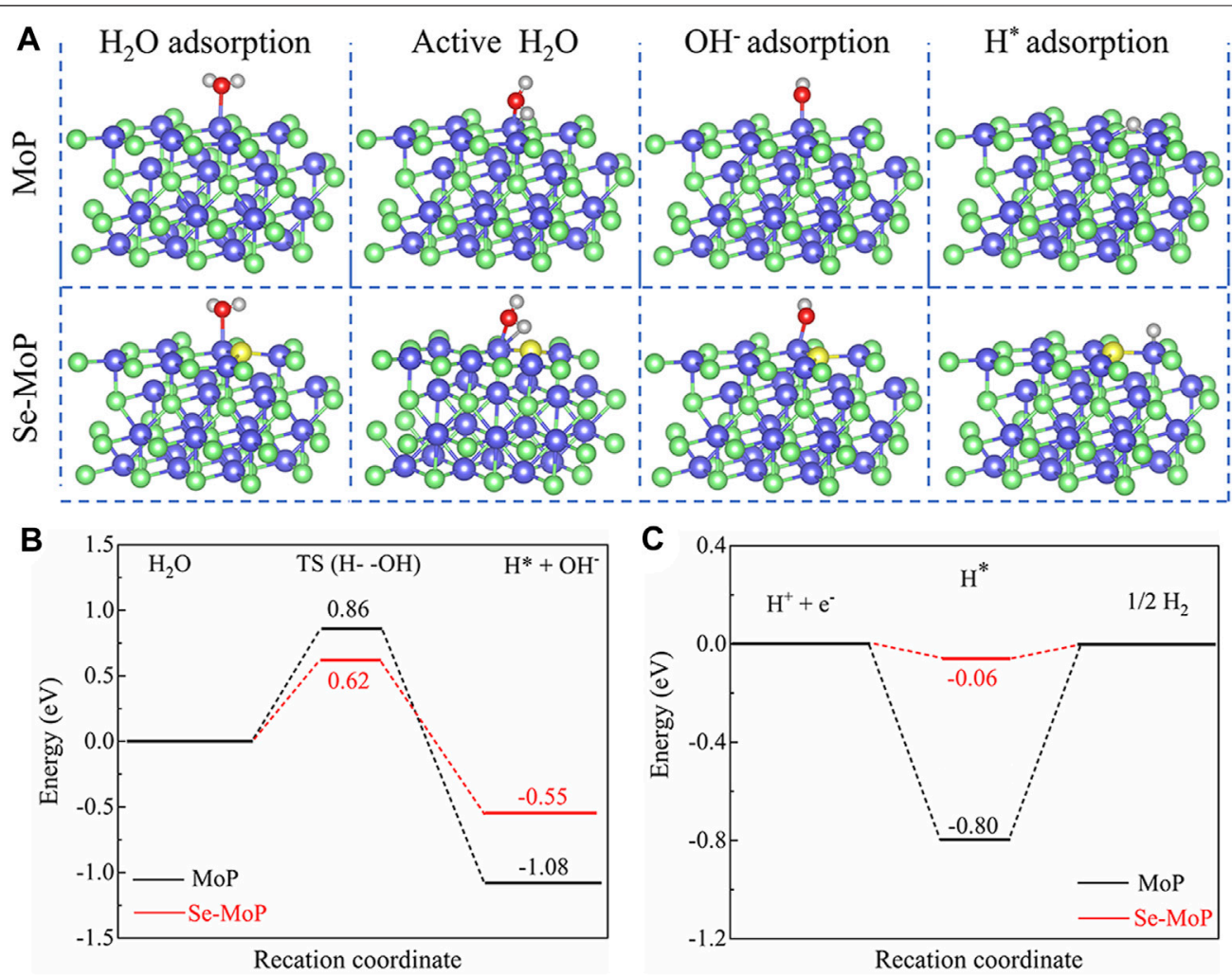

FIGURE 4 | DFT calculations of pure MoP and Se doped MoP. (A) Calculation models of the surface, $\mathrm{H}_{2} \mathrm{O}$ adsorption, $\mathrm{OH}^{-}$adsorption and $\mathrm{H}^{\star}$ adsorption of MoP and Se-MoP. The blue, green, yellow, red, and gray spheres represent Mo, $\mathrm{P}, \mathrm{Se}, \mathrm{O}$, and $\mathrm{H}$ atoms, respectively. (B) The calculated activation energy barriers of $\mathrm{H}_{2} \mathrm{O}$ molecules and $(\mathbf{C})$ adsorption energies of $\mathrm{H}^{*}$ on MoP (black) and Se-MoP surfaces (red).

nanowire. More importantly, the Se-MoP nanowires only required an overpotential of $320 \mathrm{mV}$ for delivering an industrial current density of $500 \mathrm{~mA} \mathrm{~cm}^{-2}$ (inset in Figure 3A).

As displayed in Figure $3 \mathrm{C}$, the Tafel plots of various electrocatalysts were investigated. Obviously, the Se-MoP nanowires presented a small Tafel slope of $63 \mathrm{mV} \mathrm{dec}^{-1}$, much lower than $103 \mathrm{mV} \mathrm{dec}^{-1}$ for $\mathrm{MoP}$ nanowires. As generally accepted, HER mechanism was ascribed to the following two pathways in alkaline electrolyte (Strmcnik et al., 2016): the first step is an electrochemical reduction (Volmer step: $\mathrm{H}_{2} \mathrm{O}+\mathrm{e}^{-}+{ }^{*} \rightarrow$ $\mathrm{H}^{\star}+\mathrm{OH}^{-}$) with a Tafel slop of $118 \mathrm{mV} \mathrm{dec}{ }^{-1}$. And the second step is either the ion and atom reaction (Heyrovsky step: $\mathrm{H}^{*}+\mathrm{H}_{2} \mathrm{O}+\mathrm{e}^{-}$ $\rightarrow \mathrm{H}_{2}+\mathrm{OH}^{-}+{ }^{*}$ ) or the atom combination reaction (Tafel step: $\mathrm{H}^{\star}$ $\left.+\mathrm{H}^{\star} \rightarrow \mathrm{H}_{2}+2^{\star}\right)$, corresponding to a slopes of 39 or $29 \mathrm{mV} \mathrm{dec}{ }^{-1}$, respectively. Therefore, the low slope $\left(63 \mathrm{mV} \mathrm{dec}^{-1}\right)$ on Se-MoP catalysts suggested a possible Volmer-Heyrovsky mechanism, and the $\mathrm{H}^{\star}$ desorption to gaseous $\mathrm{H}_{2}$ is the rate-determining step (Man et al., 2011). Meanwhile, the exchange current density $\left(j_{0}\right)$ of Se-MoP catalysts was determined to be $1.193 \mathrm{~mA} \mathrm{~cm}^{-2}$, which was higher than $0.251 \mathrm{~mA} \mathrm{~cm}^{-2}$ for MoP (Supplementary Figure S13), suggesting a superior electrocatalytic activity.

To probe the reason of the excellent electrocatalytic performance, the electrochemical impedance spectroscopy (EIS) of MoP and Se-MoP nanowires film was first investigated at $-0.1 \mathrm{~V}$. Based on the Nyquist plots (Figure 3E), the charge transfer resistance $\left(R_{c t}\right)$ of the Se-MoP nanowires was fitted to be $\sim 6.03 \Omega$, which was lower than $\sim 46.14 \Omega$ for MoP nanowires, indicating that the doping of Se facilitated the electron transport during the HER. Furthermore, combining with CV scans at different scan rates (Supplementary Figure S14), electrochemical double-layer capacitances $\left(C_{\mathrm{dl}}\right)$ were obtained to determine the electrochemical active surface area (ECSA), which was proportional to the ECSA (Zhu et al., 2020). As disclosed in Figure 3F, the $\mathrm{C}_{\mathrm{dl}}$ of the Se-MoP nanowires was $92.0 \mathrm{mF} \mathrm{cm}^{-2}$, which was higher than that of the MoP nanowires $\left(61.5 \mathrm{mF} \mathrm{cm}^{-2}\right)$. This result indicates that Se doping generates more exposed active sites. Then, the LSV curves was further normalized versus their ESCA (Supplementary Figure S15). Clearly, at $0.15 \mathrm{~mA} \mathrm{~cm}^{-2}$, the HER overpotential of the SeMoP nanowires was $268 \mathrm{mV}$, which was much lower than $318 \mathrm{mV}$ for the MoP nanowires. Thus, the active sites in Se$\mathrm{MoP}$ nanowires are intrinsically high-activity. The turnover frequency (TOF) is an important parameter for evaluating the inherent activity of the electrocatalysts. As illustrated in Supplementary Figure S16, the Se-MoP nanowires acquired a considerably higher TOF value of $\sim 0.229 \mathrm{~s}^{-1}$ at an overpotential of $100 \mathrm{mV}$ than $0.023 \mathrm{~s}^{-1}$ for MoP nanowires.

To investigate the influence of $\mathrm{Se}$ contents on the electrocatalytic performance of the MoP nanowires, we further assessed the electrochemical performance of different Se-MoP catalysts with different Se contents. As shown in Supplementary Figure S17A, the Se-MoP nanowires with 3.42 at\% Se afforded an 
overpotentials of $61 \mathrm{mV}$ at a current density of $10 \mathrm{~mA} \mathrm{~cm}^{-2}$ compared to $114 \mathrm{mV}$ for Se-MoP with 1.27 at\% Se and $105 \mathrm{mV}$ for Se-MoP with 5.94 at\% Se, respectively. Even under large current densities like 100 and $200 \mathrm{~mA} \mathrm{~cm}^{-2}$ (Supplementary Figure S17B), the Se-MoP nanowires with 3.42 at $\%$ Se still exhibited the smallest overpotential of $\sim 144$ and $191 \mathrm{mV}$, respectively. Therefore, the superior HER activity of the Se-MoP nanowires is ascribed to the optimized Se doping.

For assessing the electrocatalytic stability of Se-MoP catalyst, we first conducted $2000 \mathrm{CV}$ cycles between $0.025 \mathrm{~V}$ and $-0.375 \mathrm{~V}$ at a scan rate of $50 \mathrm{mV} \mathrm{s}^{-1}$. Apparently, the HER overpotential showed a negligible decline at the same current density compared to the initial state (Figure 3G). Subsequently, the long-term stability tests for $20 \mathrm{~h}$ were conducted in $1 \mathrm{M} \mathrm{KOH}$ (Figure 3L). The Se-MoP nanowires retained a steady HER overpotential over a period of $20 \mathrm{~h}$. After the long-term stability measurement, the Se-MoP nanowires was further investigated using the XRD, SEM, TEM, and the corresponding EDX mapping characterizations (Supplementary Figures S18-S20). Clearly, the structural morphology and chemical composition of Se-MoP nanowires remain well, suggesting outstanding stability during the HER process.

To gain deep insights into the enhanced mechanism of HER for Se doped MoP nanowires, density functional theory (DFT) calculations of MoP and Se-MoP were conducted (Figure 4A). We first calculated the initial water dissociation energy barriers (Volmer step). The Se-MoP showed a dramatically decreased water dissociation energy barrier $\left[\Delta G\left(\mathrm{H}_{2} \mathrm{O}\right)\right]$ of $0.62 \mathrm{eV}$, which was much lower than the value of $0.86 \mathrm{eV}$ for $\mathrm{MoP}$ (Figure 4B). Additionally, we also calculated the hydrogen adsorption free energy $\left(\Delta \mathrm{G}_{\mathrm{H}}\right)$, whose value close to $0 \mathrm{eV}$ indicated a high HER activity (Greeley et al., 2006). As shown in Figure 4C, the $\Delta \mathrm{G}_{\mathrm{H}}$ $(-0.06 \mathrm{eV})$ of $\mathrm{Se}-\mathrm{MoP}$ was much closer to $0 \mathrm{eV}$ relative to the $\Delta \mathrm{G}_{\mathrm{H}}$ of $\mathrm{MoP}(-0.80 \mathrm{eV})$. Therefore, these theoretical results demonstrate that promoting Volmer and Heyrovsky processes on Se-MoP leads to a rapid water-reduction kinetics toward excellent HER performance in alkaline electrolyte.

\section{CONCLUSION}

In summary, we synthesized a Se-doped MoP nanowire cathode through a phosphorization-selenization treatment using a Mo nanowire film as the precursor. Compared to those for preciously

\section{REFERENCES}

Anjum, M. A. R., and Lee, J. S. (2017). Sulfur and Nitrogen Dual-Doped Molybdenum Phosphide Nanocrystallites as an Active and Stable Hydrogen Evolution Reaction Electrocatalyst in Acidic and Alkaline Media. ACS Catal. 7 (4), 3030-3038. doi:10.1021/acscatal.7b00555

Blöchl, P. E. (1994). Projector Augmented-Wave Method. Phys. Rev. B 50 (24), 17953-17979. doi:10.1103/PhysRevB.50.17953

Chen, G., Wang, T., Zhang, J., Liu, P., Sun, H., Zhuang, X., et al. (2018). Accelerated Hydrogen Evolution Kinetics on NiFe-Layered Double Hydroxide Electrocatalysts by Tailoring Water Dissociation Active Sites. Adv. Mater. 30 (10), 1706279. doi:10.1002/adma.201706279 reported MoP-based electrocatalysts, the Se-MoP nanowire cathode shows greatly improved HER activity with a lower overpotential of $\sim 61 \mathrm{mV}$ at $10 \mathrm{~mA} \mathrm{~cm}^{-2}$ in alkaline solution. As revealed by the experimental results and DFT calculations, the incorporation of Se boosts water dissociation and optimizes hydrogen free energy adsorption on MoP, thus accelerating alkaline HER kinetics. The study affords a new approach for designing high-performance transition metal phosphides electrocatalysts for HER.

\section{DATA AVAILABILITY STATEMENT}

The original contributions presented in the study are included in the article/Supplementary Material, further inquiries can be directed to the corresponding authors.

\section{AUTHOR CONTRIBUTIONS}

ZL, LZ, HZ, and JZ contributed conception and design of the study and wrote sections of the manuscript. JB, WM, and BY performed the data analysis. ZL wrote the first draft of the manuscript. SL, JZ helped perform the analysis with constructive discussions. All authors contributed to manuscript revision, read, and approved the submitted version.

\section{FUNDING}

This work was supported by the Fundamental Research Funds for the Central Universities (Grant No. G2019KY05318) and the National Natural Science Foundation of China (No. 22005245, No. 51705407, No. 51674201). This work was supported by the startup fund (No. D5000210090) of Northwestern Polytechnical University. This work is partially sponsored by the Specialized Research Fund for the Doctoral Program of Higher Education (2016M600785, 2016BSHEDZZ126, 2018T111048).

\section{SUPPLEMENTARY MATERIAL}

The Supplementary Material for this article can be found online at: https://www.frontiersin.org/articles/10.3389/fchem.2021.737495/ full\#supplementary-material

Chen, N., Zhang, W., Zeng, J., He, L., Li, D., and Gao, Q. (2020). PlasmaEngineered MoP with Nitrogen Doping: Electron Localization toward Efficient Alkaline Hydrogen Evolution. Appl. Catal. B: Environ. 268, 118441. doi:10.1016/j.apcatb.2019.118441

Chen, Y.-Y., Zhang, Y., Zhang, X., Tang, T., Luo, H., Niu, S., et al. (2017). SelfTemplated Fabrication of MoNi4/MoO3-xNanorod Arrays with Dual Active Components for Highly Efficient Hydrogen Evolution. Adv. Mater. 29 (39), 1703311. doi:10.1002/adma.201703311

Chu, S., Cui, Y., and Liu, N. (2017). The Path towards Sustainable Energy. Nat. Mater 16 (1), 16-22. doi:10.1038/nmat4834

Chu, S., and Majumdar, A. (2012). Opportunities and Challenges for a Sustainable Energy Future. Nature 488 (7411), 294-303. doi:10.1038/ nature 11475 
Du, C., Shang, M., Mao, J., and Song, W. (2017). Hierarchical MoP/Ni2P Heterostructures on Nickel Foam for Efficient Water Splitting. J. Mater. Chem. A. 5 (30), 15940-15949. doi:10.1039/C7TA03669H

Fang, S., Zhu, X., Liu, X., Gu, J., Liu, W., Wang, D., et al. (2020). Uncovering Nearfree Platinum Single-Atom Dynamics during Electrochemical Hydrogen Evolution Reaction. Nat. Commun. 11 (1), 1029. doi:10.1038/s41467-02014848-2

Greeley, J., Jaramillo, T. F., Bonde, J., Chorkendorff, I., and Nørskov, J. K. (2006). Computational High-Throughput Screening of Electrocatalytic Materials for Hydrogen Evolution. Nat. Mater 5 (11), 909-913. doi:10.1038/nmat1752

Henkelman, G., Uberuaga, B. P., and Jónsson, H. (2000). A Climbing Image Nudged Elastic Band Method for Finding Saddle Points and Minimum Energy Paths. J. Chem. Phys. 113 (22), 9901-9904. doi:10.1063/1.1329672

Huang, Y., Song, X., Deng, J., Zha, C., Huang, W., Wu, Y., et al. (2019). Ultradispersed Molybdenum Phosphide and Phosphosulfide Nanoparticles on Hierarchical Carbonaceous Scaffolds for Hydrogen Evolution Electrocatalysis. Appl. Catal. B: Environ. 245, 656-661. doi:10.1016/j.apcatb.2019.01.034

Jaramillo, T. F., Jorgensen, K. P., Bonde, J., Nielsen, J. H., Horch, S., and Chorkendorff, I. (2007). Identification of Active Edge Sites for Electrochemical H2 Evolution from MoS2 Nanocatalysts. Science 317 (5834), 100-102. doi:10.1126/science.1141483

Kibsgaard, J., and Jaramillo, T. F. (2014). Molybdenum Phosphosulfide: An Active, Acid-Stable, Earth-Abundant Catalyst for the Hydrogen Evolution Reaction. Angew. Chem. Int. Ed. 53 (52), 14433-14437. doi:10.1002/anie.201408222

Kim, J., Jung, H., Jung, S.-M., Hwang, J., Kim, D. Y., Lee, N., et al. (2021). Tailoring Binding Abilities by Incorporating Oxophilic Transition Metals on 3D Nanostructured Ni Arrays for Accelerated Alkaline Hydrogen Evolution Reaction. J. Am. Chem. Soc. 143 (3), 1399-1408. doi:10.1021/jacs.0c10661

Kresse, G., and Furthmüller, J. (1996a). Efficiency of Ab-Initio Total Energy Calculations for Metals and Semiconductors Using a Plane-Wave Basis Set. Comput. Mater. Sci. 6 (1), 15-50. doi:10.1016/0927-0256(96)00008-0

Kresse, G., and Furthmüller, J. (1996b). Efficient Iterative Schemes Forab Initiototal-Energy Calculations Using a Plane-Wave Basis Set. Phys. Rev. B 54 (16), 11169-11186. doi:10.1103/PhysRevB.54.11169

Kresse, G., and Joubert, D. (1999). From Ultrasoft Pseudopotentials to the Projector Augmented-Wave Method. Phys. Rev. B 59 (3), 1758-1775. doi:10.1103/PhysRevB.59.1758

Lei, Y., Wang, Y., Liu, Y., Song, C., Li, Q., Wang, D., et al. (2020). Designing Atomic Active Centers for Hydrogen Evolution Electrocatalysts. Angew. Chem. Int. Ed. 59 (47), 20794-20812. doi:10.1002/anie.201914647

Liang, K., Pakhira, S., Yang, Z., Nijamudheen, A., Ju, L., Wang, M., et al. (2019). S-doped MoP Nanoporous Layer toward High-Efficiency Hydrogen Evolution in pH-Universal Electrolyte. ACS Catal. 9 (1), 651-659. doi:10.1021/ acscatal.8b04291

Liu, B., Li, H., Cao, B., Jiang, J., Gao, R., and Zhang, J. (2018). Few Layered N, P Dual-Doped Carbon-Encapsulated Ultrafine MoP Nanocrystal/MoP Cluster Hybrids on Carbon Cloth: An Ultrahigh Active and Durable 3D Self-Supported Integrated Electrode for Hydrogen Evolution Reaction in a Wide pH Range. Adv. Funct. Mater. 28 (30), 1801527. doi:10.1002/adfm.201801527

Liu, B., Wang, J., Mou, D., Fu, J., Chen, W., Fu, Z., et al. (2021). The Mechanism and Surface Engineering of Carbon Encapsulate Defects-Rich Molybdenum Phosphide for the Hydrogen Evolution Reaction in Alkaline media. J. Alloys Compd. 850, 156737. doi:10.1016/j.jallcom.2020.156737

Liu, Z., Zhang, G., Bu, J., Ma, W., Yang, B., Zhong, H., et al. (2020). SingleCrystalline Mo-Nanowire-Mediated Directional Growth of High-IndexFaceted MoNi Electrocatalyst for Ultralong-Term Alkaline Hydrogen Evolution. ACS Appl. Mater. Inter. 12 (32), 36259-36267. doi:10.1021/ acsami.0c11716

Ma, Y.-Y., Wu, C.-X., Feng, X.-J., Tan, H.-Q., Yan, L.-K., Liu, Y., et al. (2017). Highly Efficient Hydrogen Evolution from Seawater by a Low-Cost and Stable CoMoP@C Electrocatalyst superior to Pt/C. Energy Environ. Sci. 10 (3), 788-798. doi:10.1039/C6EE03768B

Man, I. C., Su, H. Y., Calle-Vallejo, F., Hansen, H. A., Martínez, J. I., Inoglu, N. G., et al. (2011). Universality in Oxygen Evolution Electrocatalysis on Oxide Surfaces. ChemCatChem 3 (7), 1159-1165. doi:10.1002/cctc.201000397

Norskov, J. K., and Christensen, C. H. (2006). Toward Efficient Hydrogen Production at Surfaces. Science 312 (5778), 1322-1323. doi:10.1126/ science. 1127180
Peng, L., Liao, M., Zheng, X., Nie, Y., Zhang, L., Wang, M., et al. (2020). Accelerated Alkaline Hydrogen Evolution on $\mathrm{M}(\mathrm{OH}) \mathrm{x} / \mathrm{M}-\mathrm{MoPOx}(\mathrm{M}=\mathrm{Ni}, \mathrm{Co}, \mathrm{Fe}, \mathrm{Mn})$ Electrocatalysts by Coupling Water Dissociation and Hydrogen Ad-Desorption Steps. Chem. Sci. 11 (9), 2487-2493. doi:10.1039/C9SC04603H

Perdew, J. P., Burke, K., and Ernzerhof, M. (1996). Generalized Gradient Approximation Made Simple. Phys. Rev. Lett. 77 (18), 3865-3868. doi:10.1103/PhysRevLett.77.3865

Seh, Z. W., Kibsgaard, J., Dickens, C. F., Chorkendorff, I., Nørskov, J. K., and Jaramillo, T. F. (2017). Combining Theory and experiment in Electrocatalysis: Insights into Materials Design. Science 355 (6321), eaad4998. doi:10.1126/ science.aad4998

Shi, Y., and Zhang, B. (2016). Recent Advances in Transition Metal Phosphide Nanomaterials: Synthesis and Applications in Hydrogen Evolution Reaction. Chem. Soc. Rev. 45 (6), 1529-1541. doi:10.1039/C5CS00434A

Song, H., Li, Y., Shang, L., Tang, Z., Zhang, T., and Lu, S. (2020). Designed Controllable Nitrogen-Doped Carbon-Dots-Loaded MoP Nanoparticles for Boosting Hydrogen Evolution Reaction in Alkaline Medium. Nano Energy 72, 104730. doi:10.1016/j.nanoen.2020.104730

Strmcnik, D., Lopes, P. P., Genorio, B., Stamenkovic, V. R., and Markovic, N. M. (2016). Design Principles for Hydrogen Evolution Reaction Catalyst Materials. Nano Energy 29, 29-36. doi:10.1016/j.nanoen.2016.04.017

Subbaraman, R., Tripkovic, D., Chang, K.-C., Strmcnik, D., Paulikas, A. P., Hirunsit, P., et al. (2012). Trends in Activity for the Water Electrolyser Reactions on $3 \mathrm{~d} \mathrm{M}(\mathrm{Ni}, \mathrm{Co}, \mathrm{Fe}, \mathrm{Mn}) \mathrm{Hydr}(\mathrm{oxy})$ oxide Catalysts. Nat. Mater 11 (6), 550-557. doi:10.1038/nmat3313

Turner, J. A. (2004). Sustainable Hydrogen Production. Science 305 (5686), 972-974. doi:10.1126/science.1103197

Wang, J., Gao, Y., Kong, H., Kim, J., Choi, S., Ciucci, F., et al. (2020a). Nonprecious-metal Catalysts for Alkaline Water Electrolysis: Operando Characterizations, Theoretical Calculations, and Recent Advances. Chem. Soc. Rev. 49 (24), 9154-9196. doi:10.1039/D0CS00575D

Wang, X., Gong, Y., Shi, G., Chow, W. L., Keyshar, K., Ye, G., et al. (2014). Chemical Vapor Deposition Growth of Crystalline Monolayer MoSe2. ACS Nano 8 (5), 5125-5131. doi:10.1021/nn501175k

Wang, X., Zheng, Y., Sheng, W., Xu, Z. J., Jaroniec, M., and Qiao, S.-Z. (2020b). Strategies for Design of Electrocatalysts for Hydrogen Evolution under Alkaline Conditions. Mater. Today 36, 125-138. doi:10.1016/j.mattod.2019.12.003

Xiao, P., Sk, M. A., Thia, L., Ge, X., Lim, R. J., Wang, J.-Y., et al. (2014). Molybdenum Phosphide as an Efficient Electrocatalyst for the Hydrogen Evolution Reaction. Energ. Environ. Sci. 7 (8), 2624-2629. doi:10.1039/ C4EE00957F

Xiao, W., Zhang, L., Bukhvalov, D., Chen, Z., Zou, Z., Shang, L., et al. (2020). Hierarchical Ultrathin Carbon Encapsulating Transition Metal Doped MoP Electrocatalysts for Efficient and $\mathrm{pH}-$ Universal Hydrogen Evolution Reaction. Nano Energy 70, 104445. doi:10.1016/j.nanoen.2020.104445

Xing, Z., Liu, Q., Asiri, A. M., and Sun, X. (2014). Closely Interconnected Network of Molybdenum Phosphide Nanoparticles: A Highly Efficient Electrocatalyst for Generating Hydrogen from Water. Adv. Mater. 26 (32), 5702-5707. doi:10.1002/adma.201401692

Xu, K., Ding, H., Zhang, M., Chen, M., Hao, Z., Zhang, L., et al. (2017). Regulating Water-Reduction Kinetics in Cobalt Phosphide for Enhancing HER Catalytic Activity in Alkaline Solution. Adv. Mater. 29 (28), 1606980. doi:10.1002/ adma. 201606980

Xue, S., Haid, R. W., Kluge, R. M., Ding, X., Garlyyev, B., Fichtner, J., et al. (2020). Enhancing the Hydrogen Evolution Reaction Activity of Platinum Electrodes in Alkaline Media Using Nickel-Iron Clusters. Angew. Chem. Int. Ed. 59 (27), 10934-10938. doi:10.1002/anie.202000383

Yang, J., Zhang, F., Wang, X., He, D., Wu, G., Yang, Q., et al. (2016). Porous Molybdenum Phosphide Nano-Octahedrons Derived from Confined Phosphorization in UIO-66 for Efficient Hydrogen Evolution. Angew. Chem. Int. Ed. 55 (41), 12854-12858. doi:10.1002/anie.201604315

Yao, N., Li, P., Zhou, Z., Zhao, Y., Cheng, G., Chen, S., et al. (2019). Synergistically Tuning Water and Hydrogen Binding Abilities over Co $4 \mathrm{~N}$ by Cr Doping for Exceptional Alkaline Hydrogen Evolution Electrocatalysis. Adv. Energ. Mater. 9 (41), 1902449. doi:10.1002/aenm.201902449

Ye, R., del Angel-Vicente, P., Liu, Y., Arellano-Jimenez, M. J., Peng, Z., Wang, T., et al. (2016). High-Performance Hydrogen Evolution from MoS2(1-x)PxSolid Solution. Adv. Mater. 28 (7), 1427-1432. doi:10.1002/adma.201504866 
Yin, S., Han, J., Zou, Y., Zhou, T., and Xu, R. (2016). A Highly Efficient noble Metal Free Photocatalytic Hydrogen Evolution System Containing MoP and CdS Quantum Dots. Nanoscale 8 (30), 14438-14447. doi:10.1039/C6NR00989A

Yu, F.-Y., Lang, Z.-L., Yin, L.-Y., Feng, K., Xia, Y.-J., Tan, H.-Q., et al. (2020). Pt-O Bond as an Active Site superior to Pt0 in Hydrogen Evolution Reaction. Nat. Commun. 11 (1), 490. doi:10.1038/s41467-019-14274-Z

Zhang, C., Shi, Y., Yu, Y., Du, Y., and Zhang, B. (2018a). Engineering Sulfur Defects, Atomic Thickness, and Porous Structures into Cobalt Sulfide Nanosheets for Efficient Electrocatalytic Alkaline Hydrogen Evolution. ACS Catal. 8 (9), 8077-8083. doi:10.1021/acscatal.8b02056

Zhang, J., Wang, T., Liu, P., Liu, S., Dong, R., Zhuang, X., et al. (2016). Engineering Water Dissociation Sites in MoS2 Nanosheets for Accelerated Electrocatalytic Hydrogen Production. Energ. Environ. Sci. 9 (9), 2789-2793. doi:10.1039/C6EE01786J

Zhang, X., Yu, X., Zhang, L., Zhou, F., Liang, Y., and Wang, R. (2018b). Molybdenum Phosphide/Carbon Nanotube Hybrids as $\mathrm{pH}$-Universal Electrocatalysts for Hydrogen Evolution Reaction. Adv. Funct. Mater. 28 (16), 1706523. doi:10.1002/adfm.201706523

Zhang, X., Zhou, F., Pan, W., Liang, Y., and Wang, R. (2018c). General Construction of Molybdenum-Based Nanowire Arrays for $\mathrm{pH}$-Universal Hydrogen Evolution Electrocatalysis. Adv. Funct. Mater. 28 (43), 1804600. doi:10.1002/adfm.201804600
Zhu, J., Hu, L., Zhao, P., Lee, L. Y. S., and Wong, K.-Y. (2020). Recent Advances in Electrocatalytic Hydrogen Evolution Using Nanoparticles. Chem. Rev. 120 (2), 851-918. doi:10.1021/acs.chemrev.9b00248

Conflict of Interest: The authors declare that the research was conducted in the absence of any commercial or financial relationships that could be construed as a potential conflict of interest.

Publisher's Note: All claims expressed in this article are solely those of the authors and do not necessarily represent those of their affiliated organizations, or those of the publisher, the editors and the reviewers. Any product that may be evaluated in this article, or claim that may be made by its manufacturer, is not guaranteed or endorsed by the publisher.

Copyright (C) $2021 \mathrm{Liu}, \mathrm{Bu}, \mathrm{Ma}$, Yang, Zhang, Zhong, Li and Zhang. This is an openaccess article distributed under the terms of the Creative Commons Attribution License (CC BY). The use, distribution or reproduction in other forums is permitted, provided the original author(s) and the copyright owner(s) are credited and that the original publication in this journal is cited, in accordance with accepted academic practice. No use, distribution or reproduction is permitted which does not comply with these terms. 